\title{
Cellular Uptake and Release of Intact Lactoferrin and Its Derivatives in an Intestinal Enterocyte Model of Caco-2 Cells
}

\author{
Takumi Matsuzaki, Masao Nakamura, Takehide Nogita, and Atsushi Sato* \\ School of Bioscience and Biotechnology, Tokyo University of Technology; 1404-1 Katakura, Hachioji, Tokyo 192 \\ 0982, Japan. \\ Received January 7, 2019; accepted March 13, 2019
}

\begin{abstract}
An Intact form of lactoferrin (LF) is known to be absorbed from the small intestine and transported into the blood circulation. We reevaluated the cellular uptake and release of LF using an enterocyte model of human small intestinal cells derived from the Caco-2 cell line. In contrast to a previous report, we observed that intact bovine LF was taken up into seven and 21 d-cultured Caco-2 cells and successfully released back into the culture medium, even though the human intestinal LF receptor, intelectin-1, was not immunochemically detectable. Similar observations were made for human LF and its derivatives (the N-terminal half of $\mathrm{LF}$ designated $\mathrm{N}$-lobe and $\mathrm{Fc}$ fusions). These observations regarding the uptake and release of intact LF in Caco-2 cells were consistent with in vivo observations. Therefore, we propose that the uptake and release of intact LF by Caco-2 cells should be assessed as a potential in vitro model of in vivo LF absorption in human intestines.
\end{abstract}

Key words lactoferrin; Caco-2 cell; cellular uptake; release

\section{INTRODUCTION}

Lactoferrin (LF) is an iron-binding glycoprotein that is involved in the host innate immune defense and that is found in biological fluids such as saliva, tears, blood, and milk. ${ }^{1)}$ LF possesses many biological functions, including anti-inflammatory, anti-oxidative, anti-viral, anti-microbial, anti-tumor, and immunomodulatory effects. ${ }^{1)}$ Even though LF has a molecular mass of $80 \mathrm{kDa}$, it is still absorbed intact through the small intestine and transported to the blood circulation via the lymphatic pathway. ${ }^{2,3)}$ Due to its absorption and efficacy profile, this protein is an attractive oral drug candidate.

The Caco-2 cell line is derived from human colon adenocarcinoma cells and is the most commonly used model for human enterocytes. ${ }^{4)}$ Previous studies have reported that LF can be taken up into Caco-2 cells via a clathrin-mediated endocytic process mediated by the human intestinal lactoferrin receptor (also called human intelectin-1). ${ }^{5)}$ Moreover, bovine LF (bLF) has been shown to be taken up into $10 \mathrm{~d}$-cultured Caco- 2 cells, after which degraded bLF is released back into the culture medium. ${ }^{6}$ Another study used spontaneously differentiated cell monolayers of $21 \mathrm{~d}$-cultured Caco- 2 as an in vitro model of the intestinal barrier ${ }^{4}$ and showed, using a permeability assay, that LF transported across Caco-2 monolayers was more likely to be degraded, ${ }^{7,8)}$ which was inconsistent with in vivo events. ${ }^{3)}$ In this study, to confirm the earlier published results, we have reevaluated LF uptake and release by Caco-2 cells. Unlike previous reports, ${ }^{6}{ }^{6}$ intact bLF, human LF (hLF) and its derivatives (N-lobe and Fc fusions) were found to be successfully taken up and released by Caco-2 cells. Thus, the molecular processes of LF uptake and release by Caco- 2 cells can mimic the absorption of LF by the human small intestine.

\section{MATERIALS AND METHODS}

Reagents bLF ( $>95 \%$ purity, iron content $187 \mathrm{ng} / \mathrm{mg}$ protein) was obtained from MG Nutritionals (Melbourne, Australia). Pure recombinant hLF produced in Aspergillus niger ( $>95 \%$ purity, iron content $38 \mathrm{ng} / \mathrm{mg}$ protein) was obtained from NRL Pharma Inc. (Kawasaki, Japan). Heparin was purchased from Sigma-Aldrich (9041-08-1, St. Louis, MO, U.S.A.). All reagents for the cell cultures were obtained from FUJIFILM Wako Pure Chemical Corporation (Osaka, Japan) except where otherwise indicated. Reagents for the molecular biological experiments were obtained from Toyobo (Osaka, Japan).

Cell Culture CHO DG44 (dhfr-) cells were obtained from Invitrogen (Life Technologies Japan Ltd., Tokyo, Japan) and cultured in $\alpha$-modified Eagle's medium (MEM- $\alpha$ ) with ribonucleosides and deoxyribonucleosides (Nacalai Tesque, Kyoto, Japan) supplemented with 10\% (v/v) fetal bovine serum (FBS). The human intestinal epithelial cell line Caco-2 was acquired from DS Pharma Biomedical (Osaka, Japan) or Riken BRC (RCB0988, Ibaraki, Japan). Caco-2 cells were cultured in Dulbecco's MEM (DMEM) and high glucose-supplemented with $10 \%$ (v/v) FBS, 1\% non-essential amino acids, and 1\% sodium pyruvate, with the medium being changed every three days. Note that Caco-2 cells obtained from DS Pharma Biomedical were used for all original trials. Caco-2 cells from Riken BRC were only used in secondary experiments performed to confirm the results of the primary experiment. Stable transfectants of CHO DG44 expressing human LF receptor ${ }^{9)}$ were cultured in MEM- $\alpha$ containing 10\% (v/v) FBS and without nucleosides or deoxynucleosides. The cells were maintained in $5 \% \mathrm{CO}_{2}$ at $37^{\circ} \mathrm{C}$.

Establishment of a Stable Transfectant Expressing hLF N-Lobe; Production and Purification of N-Lobe and hLFFes PCR was performed to amplify cDNA encoding human LF N-lobe (amino acids 1-351) from the plasmid pBSIILfAL ${ }^{10}$ ) using the following primers: Eco-hLF-1F, 5'-GAATTCGCC ACC ATG AAA CTT GTC TTC C-3' and Not_LFN1.2R, 5'-TCC GCG GCC GCT TAC AAG TTC TGG-3'.

* To whom correspondence should be addressed. e-mail: atsato@stf.teu.ac.jp 
A PCR fragment cleaved by EcoRI and Not I enzymes was inserted into the corresponding sites of the expression vector pOptiVecMCS. ${ }^{10)}$ The resultant plasmid was named pOptiVec/N-lobe. $d h f r$-CHO DG44 cells were then transfected with pOptiVec/N-lobe and pools of stably transfected cells were selected by a stepwise increase of methotrexate (MTX) at concentrations of $0,50,100$ and $250 \mathrm{nM}$ in dihydrofolate reductase (DHFR)-selective medium (MEM- $\alpha$ without nucleosides and deoxynucleosides). A single stable transfectant expressing high levels of N-lobe was isolated from a pool of transfectants using a limited dilution technique in MEM- $\alpha$ with $250 \mathrm{nM}$ MTX. This clone was then selected in MEM- $\alpha$ containing up to $4 \mu \mathrm{M}$ MTX $(250,500 \mathrm{nM}, 1,2$, and $4 \mu \mathrm{M})$. CHO DG44 cells producing N-lobe or hLF-Fcs (conventional hLF-hinge- $\mathrm{CH} 2-\mathrm{CH} 3^{10)}$ and hinge-deficient hLF-CH2-CH3 ${ }^{11)}$ ) were grown in Hybridoma SFM (Invitrogen). Condition media collected from the transfectants were directly subjected to purification via cation exchange chromatography using MacroCap SP (GE Healthcare Bio-Sciences KK, Tokyo, Japan), as described previously. ${ }^{10)}$

Uptake and Release of LF by Caco-2 Cells Caco-2 cells $\left(5 \times 10^{4}\right.$ cells) were seeded in a 12 -well culture plate and grown in $5 \% \mathrm{CO}_{2}$ at $37^{\circ} \mathrm{C}$ for 7 or $21 \mathrm{~d}$, as indicated. After washing the cells with phosphate buffered saline (PBS containing $137 \mathrm{mM} \mathrm{NaCl}, 2.7 \mathrm{mM} \mathrm{KCl}, 9.6 \mathrm{mM} \mathrm{Na}_{2} \mathrm{HPO}_{4}$ and $1.5 \mathrm{mM} \mathrm{KH}_{2} \mathrm{PO}_{4}$ ) three times, $0.38 \mu \mathrm{M}$ of various LFs in PBS were added and incubated for $1 \mathrm{~h}$ at 4 or $37^{\circ} \mathrm{C}$ for their uptake. Cells incubated with PBS only were used as a negative control. All cells were washed with PBS twice and then exposed to trypsin-ethylenediaminetetraacetic (EDTA) treatment for 3 min to detach them from the plates and to remove any LF attached to the cell surface. Detached cells were collected into a new tube and washed with $1 \mathrm{~mL}$ PBS three times. The presence of LFs in the third wash fraction $(12 \mu \mathrm{L})$ was checked via immunoblotting. The uptake or release of LF by Caco-2 cells was evaluated as follows:

For the analysis of LF uptake, $100 \mu \mathrm{L}$ cell lysis buffer (PBS containing $1 \%$ Triton $\mathrm{X}-100$ and a mixture of protease inhibitors [complete ${ }^{\mathrm{TM}}$, Roche Diagnostics K.K. Tokyo, Japan]) was added to the cells, which were then incubated at $4^{\circ} \mathrm{C}$ for $1 \mathrm{~h}$. The cell suspension was centrifuged at $21500 \times \boldsymbol{g}$ for $15 \mathrm{~min}$ at $4{ }^{\circ} \mathrm{C}$. The protein concentration was measured using a Bradford assay. Cell lysates ( $20 \mu \mathrm{g}$ each) were used for immunoblotting analyses.

For the analysis of released LFs taken up into Caco-2 cells, $100 \mu \mathrm{L}$ DMEM (high glucose) was added to the cells, which were then incubated at $37^{\circ} \mathrm{C}$ for the indicated time. An aliquot $(12 \mu \mathrm{L})$ of the medium was then used for the immunoblotting analysis. To examine the effect of heparin on the uptake of hLF into Caco- 2 cells, heparin with the same concentration as hLF $(0.38 \mu \mathrm{M})$ was added to the medium.

Immunoblotting A $10 \%$ polyacrylamide gel was used in sodium dodecyl sulfate-polyacrylamide gel electrophoresis (SDS-PAGE) analysis. The separated proteins were then electrotransferred onto a nitrocellulose membrane (Amersham Protran supported $0.45 \mu \mathrm{m}$ NC [GE Healthcare]) that was blocked for $1 \mathrm{~h}$ at $25^{\circ} \mathrm{C}$ in blocking buffer. Thereafter, each primary antibody at the indicated concentration was incubated at $4{ }^{\circ} \mathrm{C}$ overnight, except for horseradish peroxide (HRP)conjugated anti-human immunoglobulin $\mathrm{G}(\operatorname{IgG})(\mathrm{H}+\mathrm{L})$ ( 1 : 10000, Promega Corporation, Madison, WI, U.S.A.), which was incubated for $1 \mathrm{~h}$ at $25^{\circ} \mathrm{C}$. After washing with tris-buffered saline containing $0.05 \%$ Tween 20 (TBS-T) three times, the bound antibody was detected using an HRP-conjugated second antibody by incubating for $1 \mathrm{~h}$ at $25^{\circ} \mathrm{C}$, if necessary. The membrane was then washed with TBS-T three times. Bound antibody was detected using a sensitive luminescent reagent (Immunostar Zeta, FUJIFILM Wako Pure Chemical Corporation). Band intensities were quantified by ATTO CS analyzer 3 (ATTO, Tokyo, Japan). Conditions for immunoblotting with each antibody are summarized below.

Human LF receptor (hITLN-1): Caco-2 or CHO DG44 transfectants expressing human LF receptor ${ }^{9)}$ were lyzed in cell lysis buffer, as described above. Protein concentration was determined using the Bradford assay. Fifty micrograms of Caco-2 lysate or $10 \mu \mathrm{g}$ of CHO DG44 transfectant lysate was run on an SDS-PAGE gel under non-reducing or reducing conditions. Samples were not boiled prior to electrophoresis. TBS-T containing $2 \%$ skim milk was used as a blocking buffer. Anti-human omentin 1 antibody (Omentin 1 [human]), $\mathrm{mAb}$ [Saly-1]: 1:3000, Enzo life sciences, Farmingdale, NY, U.S.A.) and HRP-conjugated anti-mouse $\operatorname{IgG}(\mathrm{H}+\mathrm{L})$ ( 1 : 10000, Promega Corporation, Madison, WI, U.S.A.) were used as primary and secondary antibodies, respectively.

bLF: Samples were not boiled prior to electrophoresis and were run in a $10 \%$ gel under non-reducing conditions. TBS-T containing $2 \%$ skim milk was used as the blocking buffer. HRP conjugated Goat anti-bovine LF antibody (1:5000, E10126P, Bethyl laboratories Inc., Montgomery, TX, U.S.A.) was used.

hLF: Samples were not boiled prior to electrophoresis and were run in a $10 \%$ gel under non-reducing conditions. TBS-T containing $1 \%$ bovine serum albumin (BSA) was used as the blocking buffer. Human LF antibody (1:10000, A80-144A, Bethyl Laboratories, Inc.) and HRP-conjugated anti-rabbit IgG $(\mathrm{H}+\mathrm{L})(1: 10000$, Promega Corporation) were used as primary and secondary antibodies, respectively.

N-lobe: Samples were boiled prior to electrophoresis and were run in a $10 \%$ gel under reducing conditions. Reactions of primary and secondary antibodies were conducted under the

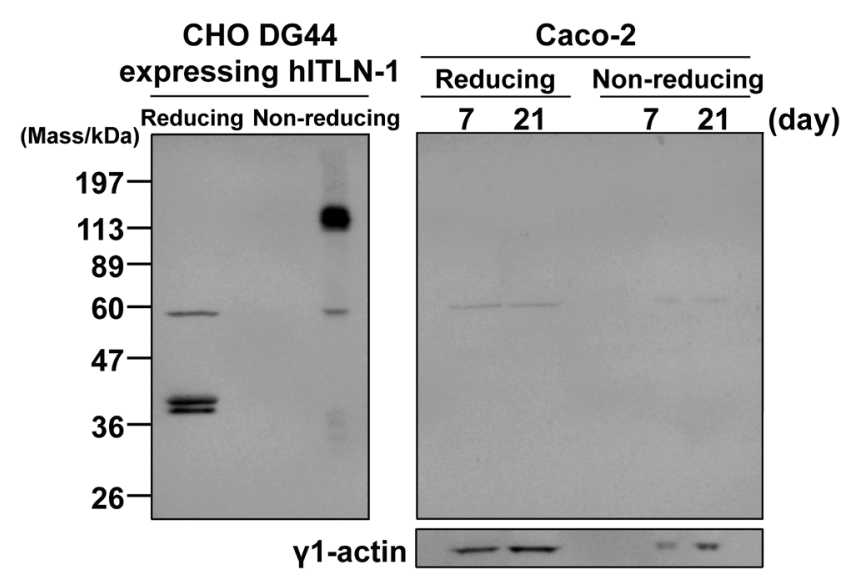

Fig. 1. Western Blot Analysis of Human Intestinal Lactoferrin Receptor (hITLN-1) Expression in Caco-2 Cells

CHO DG44-stable transfectants contained human intestinal lactoferrin receptor (hITLN-1) in its monomeric form (molecular mass of $40 \mathrm{kDa}$ ) when analyzed via Western blotting under reducing conditions, and in its trimeric form (molecular mass of $120 \mathrm{kDa}$ ) when analyzed under non-reducing conditions (left). hITLN-1 was not immunochemically detectable in Caco-2 cells cultured for 7 or $21 \mathrm{~d}$ (right). 
same conditions as described for hLF.

Human IgG Fc: Samples were not boiled prior to electrophoresis and were run in a $10 \%$ gel under non-reducing conditions. TBS-T containing $2 \%$ skim milk was used as the

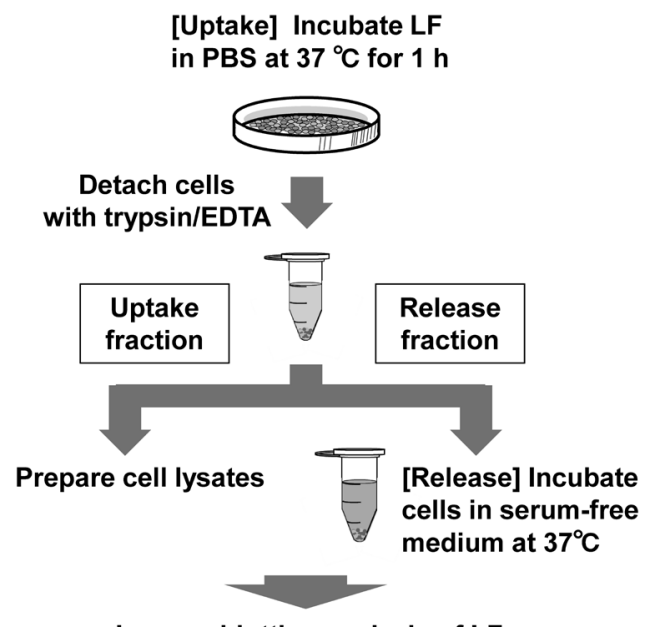

Immunoblotting analysis of LF

Fig. 2. Evaluation of LF Uptake and Release by Caco-2 Cells
A

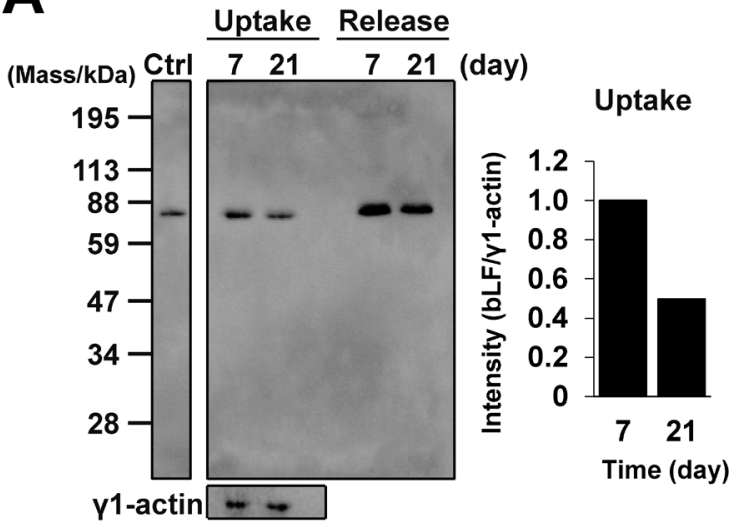

blocking buffer. HRP-conjugated Anti-Human $\operatorname{IgG}(\mathrm{H}+\mathrm{L})$ ( $1: 10000$, Promega Corporation) was added prior to incubation for $1 \mathrm{~h}$ at $25^{\circ} \mathrm{C}$.

Human $\gamma 1$ actin: The amount of $\gamma 1$ actin in cell lysates was used to show the equivalence of protein loading. After immunoblotting, the antibodies on blot membranes were removed using stripping buffer (FUJIFILM Wako Pure Chemical Corporation) followed by incubation for $10 \mathrm{~min}$ at $25^{\circ} \mathrm{C}$. Membranes were washed with TBS-T three times and blocked with TBS-T containing $1 \%$ BSA. Human anti- $\gamma 1$-actin antibody (1:5000, FUJIFILM Wako Pure Chemical Corporation) and HRP-conjugated anti-mouse IgG $(\mathrm{H}+\mathrm{L})(1: 10000$, Promega Corporation) were used as primary and secondary antibodies, respectively.

\section{RESULTS AND DISCUSSION}

Loss of Human LF Receptor Expression in 7 and $21 \mathrm{~d}$ Cultured Caco-2 Cells First, we attempted to confirm the expression of human intestinal LF receptor (referred to as hITLN-1) in Caco-2 cells cultured for $7 \mathrm{~d}$ (D7) and $21 \mathrm{~d}$ (D21) via Western blotting, since previous reports have shown that $16 \mathrm{~d}$-cultured cells express hITLN-1 and take up LF via
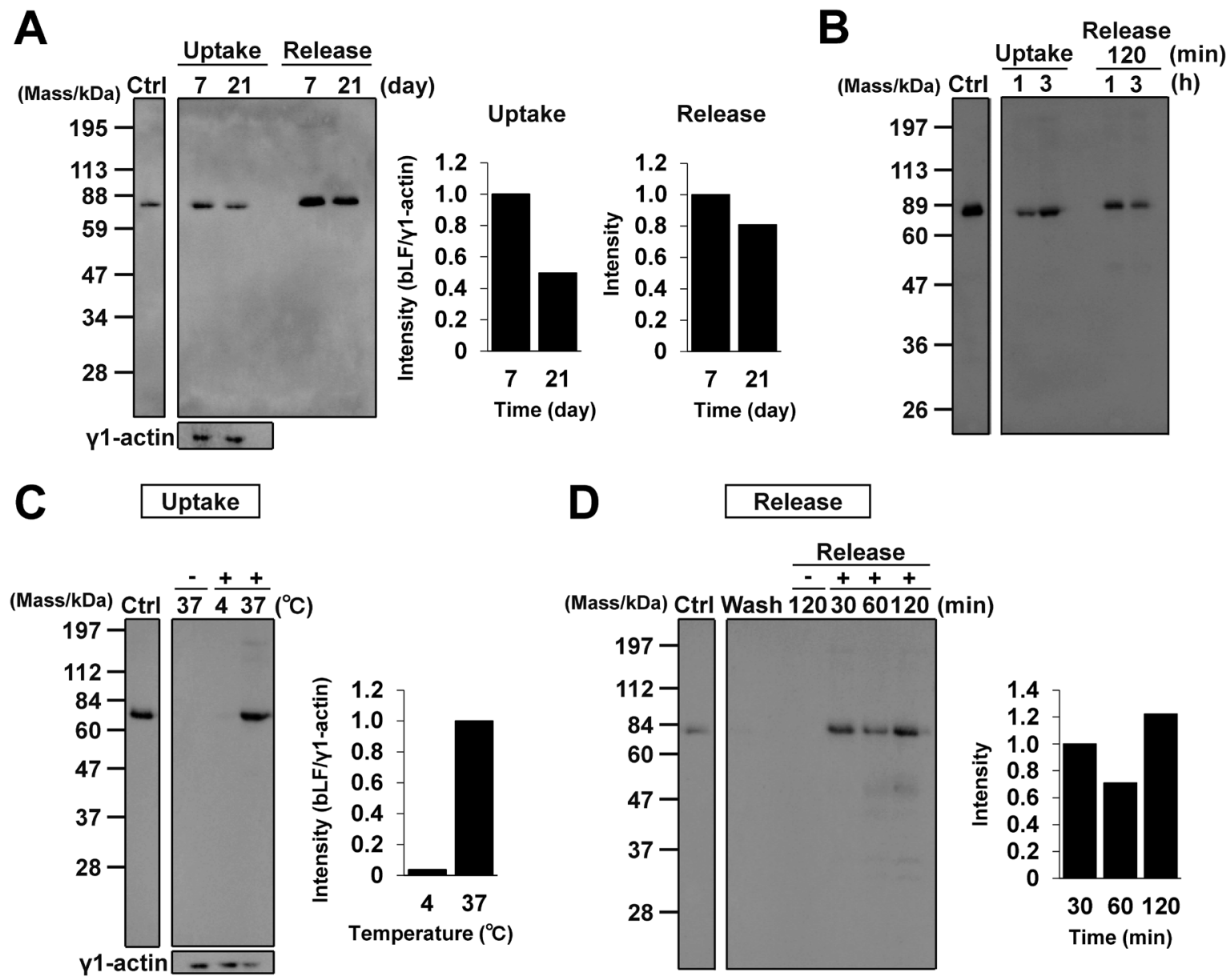

Fig. 3. Intact Bovine Lactoferrin (bLF) Taken Up into Caco-2 Cells Was Later Successfully Released Back into the Culture Medium

(A) bLF as an intact molecule was taken up from PBS by Caco-2 cells cultured for both 7 and $21 \mathrm{~d}$, and was subsequently released into the culture medium. (B) Intact bLF was also taken up from the medium (DMEM) by D7 cells with an incubation time of 1 and $3 \mathrm{~h}$, and was later successfully released into the culture supernatant during the release period for $120 \mathrm{~min}$. (C) The temperature-dependent uptake of bLF into Caco-2 cells. Cells incubated with PBS-only at $37^{\circ} \mathrm{C}$ did not show any signal (-, left). (D) Release of intact bLF into the culture medium after being taken up by Caco-2 cells. Cells incubated with PBS- only for 120 min ( - ) did not show any signal in the release step. No bLF was detected in the wash fraction. (B)-(D) Control bLF (10 ng) was detected via immunoblotting (Ctrl, left panel). Relative band intensities are represented graphically in $\mathrm{A}, \mathrm{C}$ and $\mathrm{D}$ (right). 


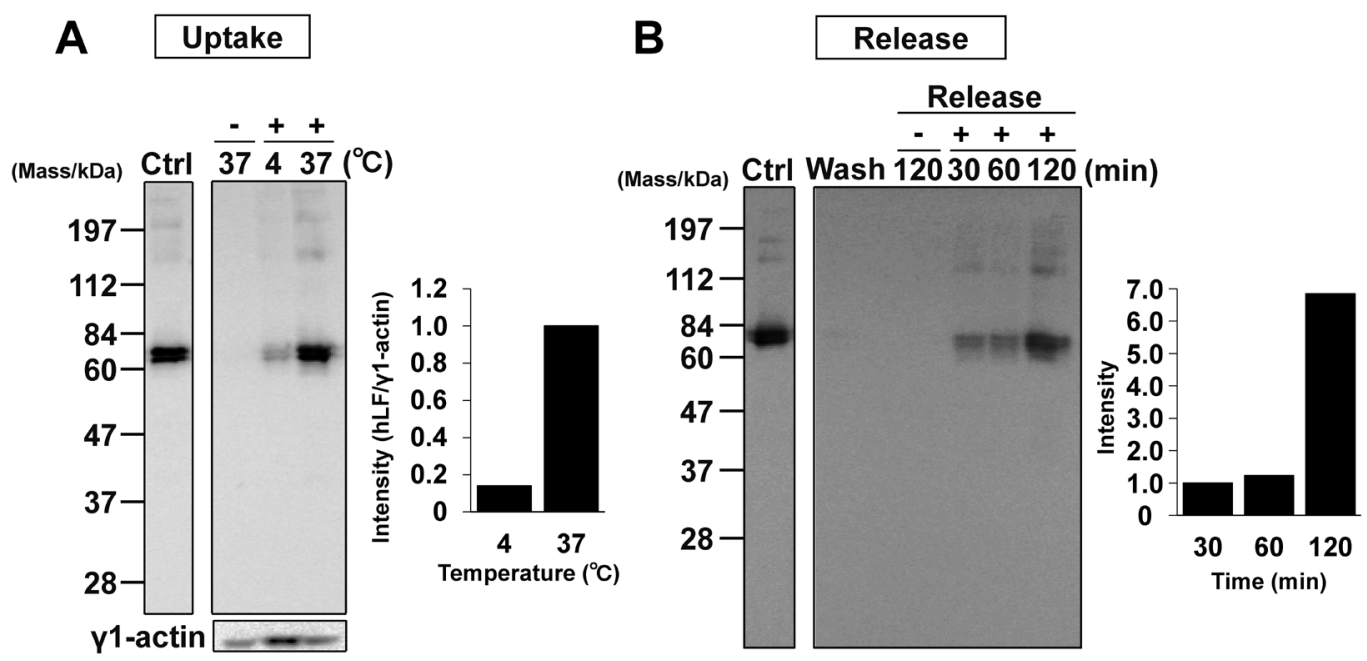

Fig. 4. Intact Human Lactoferrin (hLF) Taken Up into Caco-2 Cells Was Successfully Released into the Culture Medium

(A) The temperature-dependent uptake of hLF into Caco-2 cells. Cells incubated with PBS-only at $37^{\circ} \mathrm{C}$ did not show any signal (-, left). (B) Release of intact hLF into the culture medium after being taken up by Caco-2 cells. Cells incubated with PBS-only for $120 \mathrm{~min}(-)$ did not show any signal in the release step. No hLF was detected in the wash fraction. (A) and (B) Control hLF (10 ng) was detected via immunoblotting (Ctrl, left panel). Relative band intensities are represented graphically (right).

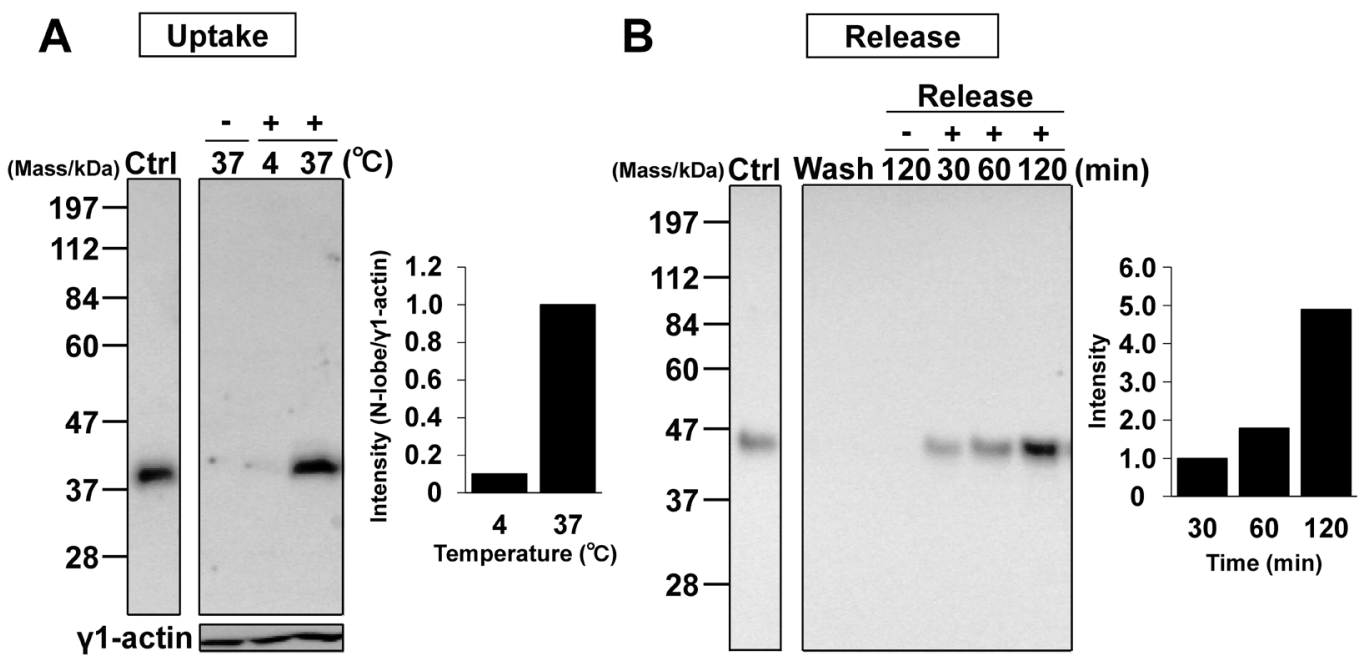

Fig. 5. Intact Human Lactoferrin (hLF) N-Lobe Taken Up into Caco-2 Cells Was Successfully Released into the Culture Medium

(A) The temperature-dependent uptake of hLF N-lobe into Caco-2 cells. Cells incubated with PBS-only at $37^{\circ} \mathrm{C}$ did not show any signal (-, left). (B) Release of intact hLF N-lobe into the culture medium after being taken up by Caco-2 cells. Cells incubated with PBS-only for $120 \mathrm{~min}$ ( - ) did not show any signal in the release step. No N-lobe was detected in the wash fraction. (A) and (B) Control hLF N-lobe (10 ng) was detected via immunoblotting (Ctrl, left panel). Relative band intensities are represented graphically (right).

hITLN-1-mediated endocytosis. ${ }^{5)}$ As a positive control, $\mathrm{CHO}$ DG44 transfectants expressing hITLN-1 ${ }^{9)}$ were analyzed, and showed a monomeric hITLN-1 (molecular mass of $40 \mathrm{kDa}$ ) under reducing conditions and a trimeric hITLN-1 (molecular mass of $120 \mathrm{kDa}$ ) under non-reducing conditions (Fig. 1). Unfortunately, hITLN-1 could not be immunochemically detected either in D7 or D21 Caco-2 cells (Fig. 1). This phenomenon was verified using cells obtained from a different source (Riken BRC, data not shown). The discrepant results seen here might be ascribed to cell culture conditions which affect Caco-2 cells differentiation.

After bLF Endocytosis, D7 and D21 Caco-2 Cells Released Intact bLF into the Culture Medium It has been reported that $1.25 \mu \mathrm{M}$ bLF in the medium (DMEM) was taken up by Caco- 2 cells cultured on a flat-bottom plate for $10 \mathrm{~d}$, after which degraded bLF was released back into the culture medium. ${ }^{6}$ We next examined the uptake of bLF (suspended in
PBS, pH 7.4) by Caco-2 cells, according to the protocol shown in Fig. 2. Unlike the previously reported results, ${ }^{6)}$ bLF as an intact molecule was taken up from PBS by both D7 and D21 cells and subsequently released from these cells into the culture medium (Fig. 3A), even though the hITLN-1 receptor was not immunochemically detectable. Intact bLF was also taken up from the medium (DMEM) by D7 cells with an incubation time of 1 and $3 \mathrm{~h}$, and was successfully released into the culture supernatant (Fig. 3B). For further analysis, we selected PBS for the cellular uptake of LF because similar results were obtained with both PBS and the medium (DMEM).

Temperature dependence of bLF uptake into D7 cells was observed (Fig. 3C). After its endocytosis into the D7 cells, bLF was released into the culture medium as an intact molecule, as was seen from the fact that neither the wash fraction nor the culture medium of D7 cells itself contained bLF (Fig. $3 \mathrm{D})$. This phenomenon was also verified with cells provided 

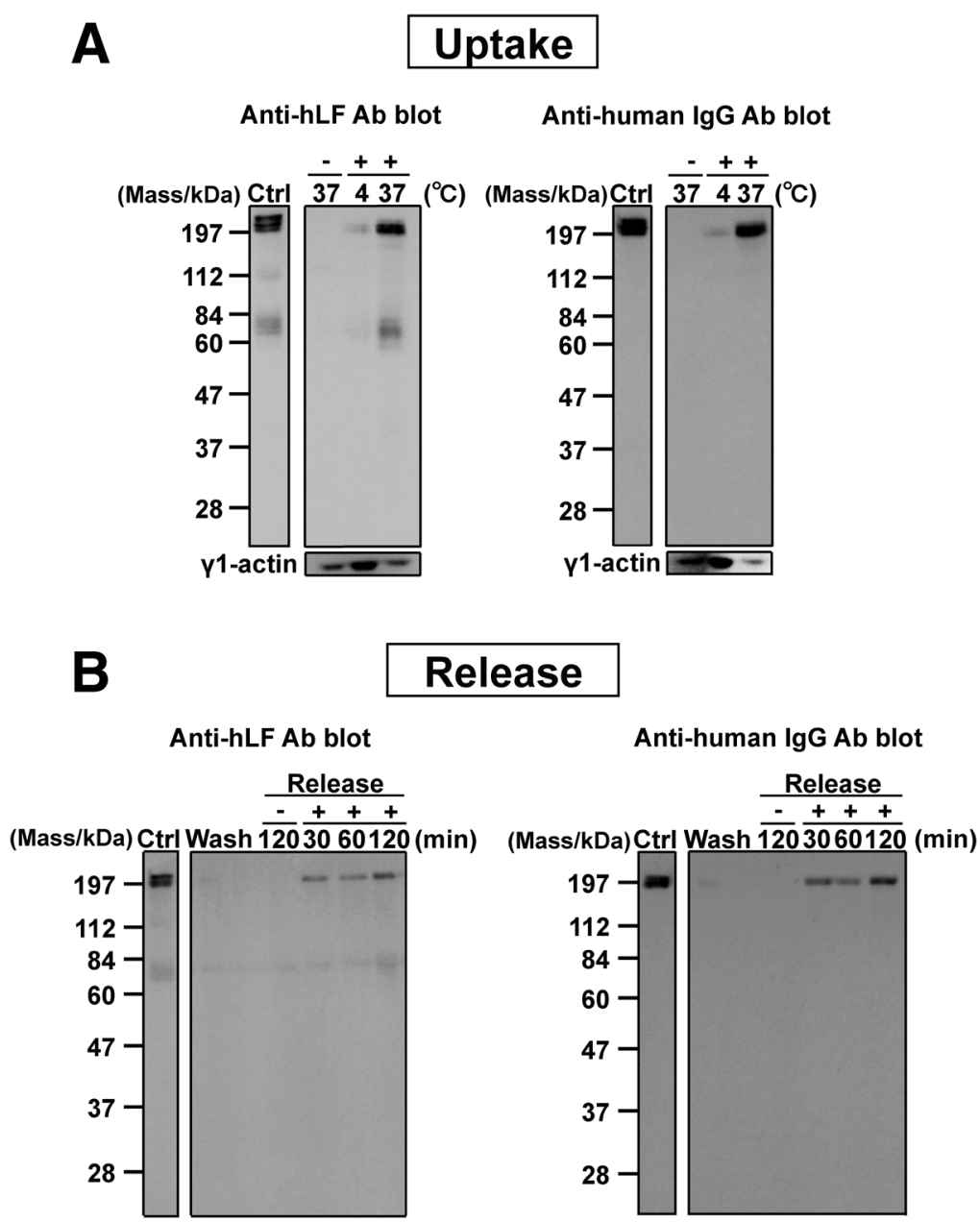

Fig. 6. Intact hLF-Hinge-CH2-CH3 Was Taken Up and Then Released by Caco-2 Cells

(A) The temperature-dependent uptake of hLF-hinge-CH2-CH3 into Caco-2 cells. Cells incubated with hLF-hinge-CH2-CH3 showed uptake of intact hLF-hinge-CH2$\mathrm{CH} 3$ in a temperature-dependent manner (immunoblotting with polyclonal anti-hLF antibody [left] and anti-human IgG (H $+\mathrm{L}$ ) antibody [right]). Cells incubated with PBS-only at $37^{\circ} \mathrm{C}$ did not show any signal (-, left). (B) Intact hLF-hinge-CH2-CH3 taken up into Caco-2 cells was later successfully released into the culture medium (immunoblotting with polyclonal anti-hLF antibody [left] and anti-human IgG (H + L) antibody [right]). Cells incubated with PBS-only for 120 min ( -$)$ did not show any signal in the release step. No hLF-hinge-CH2-CH3 was detected in the wash fraction. (A) and (B) Control hLF-hinge-CH2-CH3 (26ng) was detected via immunoblotting (Ctrl, left panel).

by Riken BRC (data not shown). Thus, D7 Caco-2 cells were used for further experiments due to the facile preparation of the cells.

Intact hLF and Its N-Lobe Were Taken up into Caco-2 Cells and Then Released into the Culture Medium Like bLF, intact hLF was also taken up into Caco-2 cells in a temperature-dependent fashion (Fig. 4A). Further, after its internalization, $\mathrm{hLF}$ was released intact into the culture medium (Fig. 4B). We have already reported that fluorescent-labeled hLF is taken up into Caco-2 cells in an ATP-dependent manner due to the inhibitory effects on its uptake by an ATP-synthesis inhibitor, sodium azide. ${ }^{10,11)}$ It is well known that ATP is required for receptor-mediated endocytosis. ${ }^{12)}$ Thus, a putative receptor or receptor-like molecule other than hITLN-1 might be involved in hLF internalization into Caco- 2 cells.

Next, the uptake and release of the N-terminal half of hLF (N-lobe) by Caco-2 cells was examined, since N-lobe has been reported as the structural unit responsible for the internalization of hLF into Caco-2 cells. ${ }^{13)}$ The results showed that hLF $\mathrm{N}$-lobe (molecular mass of $40 \mathrm{kDa}$ ) taken up by Caco- 2 cells was released intact back to the culture medium (Figs. 5A, B).

hLF-Fc Fusion Endocytosis and Subsequent Release from Caco-2 Cells Recently, we developed two long-acting hLF-Fc fusions, a conventional hLF-hinge- $\mathrm{CH} 2-\mathrm{CH}^{10)}$ and a hinge-deficient hLF-CH2-CH3. ${ }^{11)}$ Here, we examined their endocytosis and subsequent release from Caco-2 cells for their potential use as oral drugs. Intact hLF-hinge- $\mathrm{CH} 2-\mathrm{CH} 3$ was taken up into Caco-2 cells and successfully released into the culture supernatant (Figs. 6A, B). Although some degraded hLF-CH2-CH3 was found in the release fraction, intact hLF$\mathrm{CH} 2-\mathrm{CH} 3$ internalized into Caco-2 cells was also found to be released into the culture medium (Figs. 7A, B). Degraded fragments with molecular masses of approx. $80 \mathrm{kDa}$ (similar to $\mathrm{hLF}$ ) were detectable with anti-hLF antibodies but not with anti-human $\operatorname{IgG}$ antibodies. It is possible that the spacer sequence flanked by hLF and $\mathrm{CH} 2-\mathrm{CH} 3$ may have been be partially degraded (Fig. 7B). The uptake of conventional and hinge-deficient hLF-Fc fusions by Caco-2 cells in PBS (pH 7.4) was mainly mediated by hLF due to relatively poor human IgG uptake into Caco-2 cells at $\mathrm{pH}$ 7.4. ${ }^{14)}$ Further, the uptake of fluorescent-labeled hLF-Fc fusions into Caco-2 cells was completely abolished by the addition of excess amounts of unlabeled $\mathrm{bLF},{ }^{10,11)}$ which also indicated that $\mathrm{hLF}$ was mainly involved in the endocytosis of hLF-Fc fusions. 
A

\section{Uptake}

Anti-hLF Ab blot

Anti-human IgG Ab blot
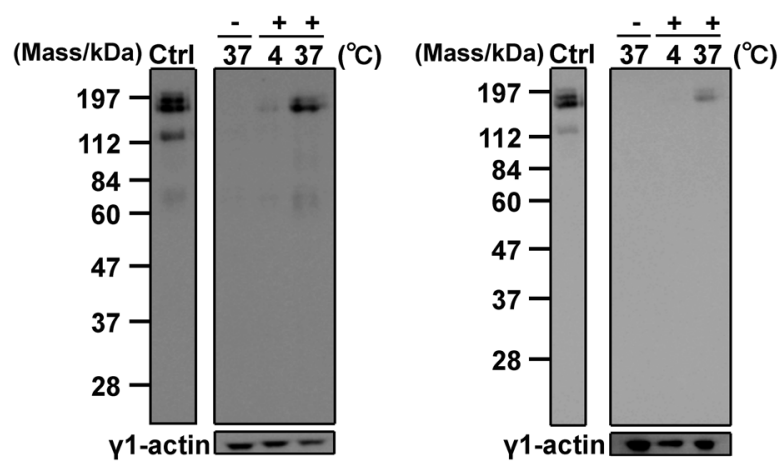

B

\section{Release}

Anti-hLF Ab blot $\frac{\text { Release }}{-++++}$

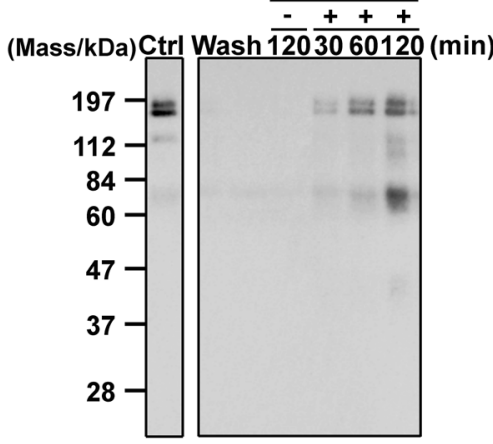

Anti-human IgG Ab blot

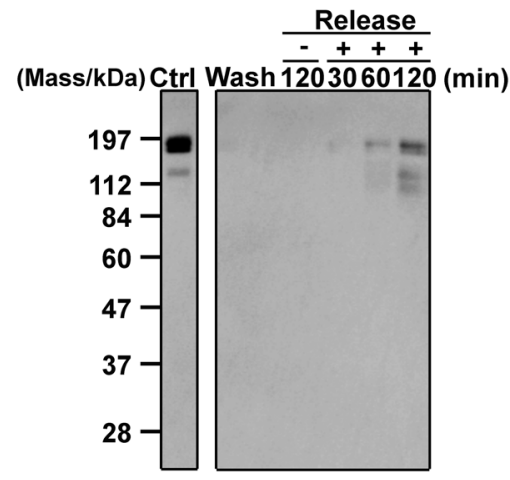

Fig. 7. Intact hLF-CH2-CH3 Taken up by Caco-2 Cells Was Then Released into the Culture Medium with Slight Degradation

(A) The temperature-dependent uptake of hLF-CH2-CH3 into Caco-2 cells. Cells incubated with hLF-CH2-CH3 showed uptake of intact hLF-CH2-CH3 in a temperature-dependent manner (immunoblotting with polyclonal anti-hLF antibody [left] and anti-human IgG $(\mathrm{H}+\mathrm{L})$ antibody [right]). Cells incubated with PBS-only at $37^{\circ} \mathrm{C}$ did not show any signal ( - left). (B) hLF-CH2-CH3 taken up into Caco-2 cells was released into the culture medium along with its degradate (immunoblotting with polyclonal anti-hLF antibody [left] and anti-human $\mathrm{IgG}(\mathrm{H}+\mathrm{L})$ antibody [right]). Cells incubated with PBS-only for $120 \mathrm{~min}(-)$ did not show any signal in the release step. No hLF-CH2-CH3 was detected in the wash fraction. (A) and (B) Control hLF-CH2-CH3 (26ng) was detected via immunoblotting (Ctrl, left panel).

Interaction between LF and Heparin Blocks the Uptake of $\mathbf{h L F}$ It has been reported that LF interacts with heparan sulfate present on the surface of most cells, since heparinase treatment $^{15)}$ or the addition of heparin has led to the attenuated binding of LF to some cells. ${ }^{16)}$ Here, we examined the effects of heparin on the uptake of hLF into Caco-2 cells. As reported previously, ${ }^{17)}$ the addition of heparin at an equimolar concentration of hLF prevented the uptake of hLF into Caco-2 cells (Fig. 8), suggesting that the heparan sulfate-recognizing sequence on hLF is required for its internalization, or alternatively, that heparan sulfate proteoglycans on Caco-2 cells might act as endocytosis receptors. ${ }^{18)}$

Taken together, unlike the previous report, ${ }^{6)}$ our study shows that intact LF and its derivatives taken up by Caco-2 cells were released extracellularly, consistent with in vivo observations. ${ }^{3)}$ So far, the possible causes for the discrepant results are not certain. Due to the heterogeneous features of the Caco-2 cell line, ${ }^{19)}$ different culture-related conditions or sources of cell clones might yield inconsistent results. However, Caco-2 cells from the two different sources (DS Pharma Biomedical and Riken BRC) both exhibited reproducible results regarding the uptake and release of bLF and hLF.

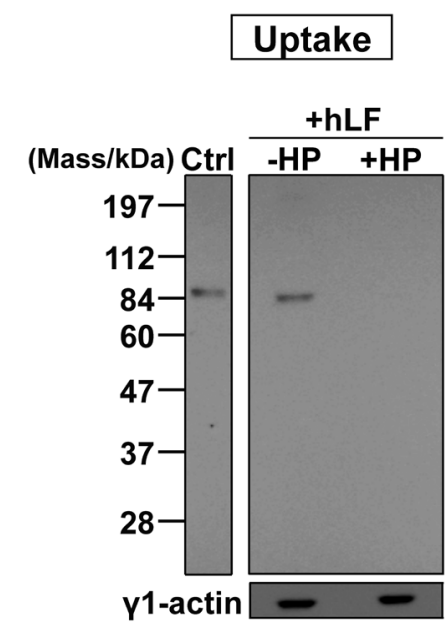

Fig. 8. Heparin Inhibits Human Lactoferrin (hLF) Endocytosis into Caco-2 Cells Due to Its Binding

HP: Heparin added at an equimolar concentration of hLF $(0.38 \mu \mathrm{M})$. Control hLF levels $(10 \mathrm{ng})$ detected via immunoblotting (Ctrl, left panel). 
The Caco-2 monolayers have been widely used as an in vitro model of the intestinal barrier to study the intestinal absorption of drugs. ${ }^{4)}$ This cell culture system could be a valuable approach to examine the intestinal absorption of LF. However, this assay can often be laborious and time-consuming. Thus, the simple assay to assess its uptake and release by Caco- 2 cells could be a potential alternative approach to evaluate the intestinal absorption of LF.

In conclusion, the simple uptake and release of intact LF by intestinal epithelial Caco-2 cells shows its potential for use as an in vitro model for the intestinal absorption of LF.

Acknowledgments We thank Mr. Y. Yanagi for technical assistance.

Conflict of Interest The authors declare no conflict of interest.

\section{REFERENCES}

1) Moreno-Expósito L, Illescas-Montes R, Melguizo-Rodriguez L, Ruiz C, Ramos-Torrecillas J, de Luna-Bertos E. Multifunctional capacity and therapeutic potential of lactoferrin. Life Sci., 195, 61-64 (2018)

2) Takeuchi T, Kitagawa H, Harada E. Evidence of lactoferrin transportation into blood circulation from intestine via lymphatic pathway in adult rats. Exp. Physiol., 89, 263-270 (2004).

3) Harada E, Itoh Y, Sitizyo K, Takeuchi T, Araki Y, Kitagawa H. Characteristic transport of lactoferrin from the intestinal lumen into the bile via the blood in piglets. Comp. Biochem. Physiol. A Mol. Integr. Physiol., 124, 321-327 (1999).

4) Smetanová L, Stětinová V, Svoboda Z, Kvetina J. Caco-2 cells, biopharmaceutics classification system (BCS) and biowaiver. Acta Medica (Hradec Kralove), 54, 3-8 (2011).

5) Jiang R, Lopez V, Kelleher SL, Lonnerdal B. Apo- and hololactoferrin are both internalized by lactoferrin receptor via clathrinmediated endocytosis but differentially affect ERK-signaling and cell proliferation in Caco-2 cells. J. Cell. Physiol., 226, 3022-3031 (2011).

6) Akiyama Y, Oshima K, Shin K, Wakabayashi H, Abe F, Nadano $\mathrm{D}$, Matsuda T. Intracellular retention and subsequent release of bovine milk lactoferrin taken up by human enterocyte-like cell lines, Caco-2, C2BBe1 and HT-29. Biosci. Biotechnol. Biochem., 77, 1023-1029 (2013).
7) Conesa C, Pocovi C, Perez MD, Calvo M, Sanchez L. Recombinant human lactoferrin and iron transport across Caco-2 monolayers: effect of heat treatment on the binding to cells. J. Agric. Food Chem., 56, 2831-2837 (2008).

8) Conesa C, Pocovi C, Perez MD, Calvo M, Sanchez L. Transport of iron bound to recombinant human lactoferrin from rice and iron citrate across Caco-2 cell monolayers. Biosci. Biotechnol. Biochem., 73, 2615-2620 (2009)

9) Oshima Y, Seki K, Shibuya M, Naka Y, Yokoyama T, Sato A. Soluble human intestinal lactoferrin receptor: $\mathrm{Ca}^{2+}$-dependent binding to sepharose-based matrices. Biol. Pharm. Bull., 39, 435-439 (2016).

10) Shiga Y, Oshima Y, Kojima Y, Sugimoto A, Tamaki N, Murata D, Takeuchi T, Sato A. Recombinant human lactoferrin-Fc fusion with an improved plasma half-life. Eur. J. Pharm. Sci., 67, 136-143 (2015).

11) Shiga $Y$, Murata D, Sugimoto A, Oshima $Y$, Tada M, Ishii-Watabe A, Imai K, Tomii K, Takeuchi T, Kagaya S, Sato A. Hinge-deficient IgG1 Fc fusion: application to human lactoferrin. Mol. Pharm., 14, 3025-3035 (2017).

12) Schmid SL, Carter LL. ATP is required for receptor-mediated endocytosis in intact cells. J. Cell Biol., 111, 2307-2318 (1990).

13) Suzuki YA, Wong H, Ashida KY, Schryvers AB, Lonnerdal B. The N1 domain of human lactoferrin is required for internalization by caco-2 cells and targeting to the nucleus. Biochemistry, 47, 10915-10920 (2008).

14) Sato K, Nagai J, Mitsui N, Yumoto R, Takano M. Effects of endocytosis inhibitors on internalization of human IgG by Caco-2 human intestinal epithelial cells. Life Sci., 85, 800-807 (2009).

15) Ji ZS, Mahley RW. Lactoferrin binding to heparan sulfate proteoglycans and the LDL receptor-related protein. Further evidence supporting the importance of direct binding of remnant lipoproteins to HSPG. Arterioscler. Thromb., 14, 2025-2031 (1994).

16) Ishii $T$, Ishimori $H$, Mori $K$, Uto $T$, Fukuda $K$, Urashima $T$, Nishimura M. Bovine lactoferrin stimulates anchorage-independent cell growth via membrane-associated chondroitin sulfate and heparan sulfate proteoglycans in PC12 cells. J. Pharmacol. Sci., 104, 366-373 (2007).

17) Ashida K, Sasaki H, Suzuki YA, Lonnerdal B. Cellular internalization of lactoferrin in intestinal epithelial cells. Biometals, 17, 311-315 (2004)

18) Christianson HC, Belting M. Heparan sulfate proteoglycan as a cell-surface endocytosis receptor. Matrix Biol., 35, 51-55 (2014).

19) Sambuy Y, De Angelis I, Ranaldi G, Scarino ML, Stammati A, Zucco F. The Caco-2 cell line as a model of the intestinal barrier: influence of cell and culture-related factors on Caco-2 cell functional characteristics. Cell Biol. Toxicol., 21, 1-26 (2005). 